\title{
2nd year doctor of physical therapy students less proficient at goniometry than the reported norm
}

\begin{abstract}
To examine the reliability of Doctor of Physical Therapy students at performing goniometric measurements of shoulder ROM using a repeated measures trial. Twenty-two volunteers were selected via a convenience sampling of $2^{\text {nd }}$ year Doctor of Physical Therapy students. The examiners were asked to take measurements of shoulder ROM using both the established method of goniometry and a novel method using innovative landmarks to determine if it results in better reliability than the standard method. Twenty-two healthy subjects were measured for bilateral shoulder flexion and abduction. Two weeks later the same examiners repeated the measurements on the same subjects. Statistical analysis of the data was completed using SPSS. ICC values were used to compare both intra-rater and inter-rater reliability. Analysis was completed for the measurement values of bilateral shoulder flexion and shoulder abduction. Ranges of ICC values were found for each data set and then compared to previously published ranges for clinicians. The results of data analysis showed large ranges for both methods. No statistical significance was found between the standard and novel method of measurement. When compared to published research, it was found that our values for both methods were much lower and inconsistent than those of the standard method in the available literature. Our results show dramatically less reliability in goniometry measurements among Doctor of Physical Therapy students than the previously published results indicate for practicing physical therapists. This may indicate the need for more instruction and more practice in the skill of goniometry for students enrolled in DPT programs.
\end{abstract}

\author{
Volume 4 Issue 4 - 2019
}

\section{Jake Irwin, Daniela Espino, Caitlyn Matthews, Todd Scott}

Department of Physical Therapy, Byrdine F Lewis School of Nursing and Health Professions, Georgia State University, USA

Correspondence: Jake Irwin, Byrdine F Lewis School of Nursing and Health Professions, Georgia State University, USA, Tel 404-4I31212, Email jirwin5@gsu.edu

Received: July 18, 2019 | Published: July 22, 2019

Keywords: Goniometry, shoulder range of motion, physical therapy

\section{Introduction}

Objective assessments, such as range of motion measurements, are extremely important to the physical therapist's ability to improve the overall health of his or her patient. Objective data allows the therapist to gain an evidence-based assessment of the patient's current level of health, as well as the ability to track improvements and the effectiveness of treatments. A vital component of these measurements is reliability. Reliability, as it relates to this topic, is defined by the New Oxford American Dictionary as "the degree to which the result of a measurement, calculation or specification can be depended upon to be accurate." In the field of physical therapy, the benefit of having a reliable process is that you can compare the measurements of multiple clinicians with confidence. Further, with a reliable process it does not matter if more than one clinician ends up treating the patient, since all measurements can be counted on to relate well to all the others.

The present study sought to validate the effectiveness of teaching a novel method of measuring shoulder range of motion to novice clinicians. $2^{\text {nd }}$ year DPT student volunteers were enrolled as clinicians to test the hypothesis that the inter- and intra-rater reliability of joint range of motion assessment will increase with the use of a novel method to measure shoulder range of motion versus the traditional method commonly used in physical therapy practice today. Measurements were taken for two shoulder motions bilaterally for both methods. If this hypothesis is validated, studies that follow may assess the use of other non-traditional methods of measuring range of motion at different joints throughout the body.

\section{Review of literatue}

Shishani, Flocken and Gobezie, in What Is the Accuracy of Shoulder Range of Motion Measurements on Physical Exam ${ }^{2}$, also compared two methods of measuring shoulder range of motion with one being a standard universal goniometer. In this study participants were measured for shoulder flexion and external rotation only. Intrarater reliability was found using ICC 2,1 . The authors found an intrarater reliability of 0.83 for the standard goniometer.

Kolber \&Hanney, ${ }^{1}$ in The Reliability and Concurrent Validity of Shoulder Mobility Measurement Using a Digital Inclinometer and Goniometer: A Technical Report. ${ }^{1}$ examined the intrarater reliability and concurrent validity of two methods of assessing shoulder mobility, one being the standard 12 " plastic goniometer. Participants were measured for flexion, abduction, external rotation and internal rotation of the shoulder of their dominant arm. Intrarater reliability was ascertained using ICC model $3, \mathrm{k}$. The authors found outstanding intrarater reliability of ${ }^{3} 0.94$ for the standard goniometer.

In Jette et al. ${ }^{2}$ in Clinical Instructor's Perceptions of Behaviors That Comprise Entry-Level Clinical Performance in Physical Therapy Students: A Qualitative Study ${ }^{2}$ sought to determine the essential skills and behaviors that Clinical Instructors deem essential to physical therapy students reaching entry-level performance. 21 physical therapists who had previous experience as CI's were interviewed to establish their idea of the necessary skill-set that an entry-level physical therapist should possess. One of the Clinical Skills reported by the participants is the ability to take range of motion measurements. 
This existing literature points to the importance of possessing the ability to perform accurate and skilled goniometric measurements for the entry-level physical therapist. The literature also establishes a benchmark intrarater reliability expectation for entry-level PT's.

\section{Experimental section}

\section{Participants}

Twenty-two examiners, 11 males and 11 females were selected via a volunteer convenience sampling of $2^{\text {nd }}$ year Doctor of Physical Therapy students at Georgia State University to act as clinicians. Twenty-two subjects were selected via convenience sample to have their shoulder ROM measured in the experiment. All $2^{\text {nd }}$ year Georgia State Physical Therapy students were invited to be examiners for this study. Exclusion criteria for this study were subjects with a current or previous shoulder pathology. The study was approved by the Institutional Review Board at Georgia State University and Informed Consent was obtained from all participants.

\section{Measurement Methods}

Two trials of data collection were completed with two weeks separating the trials. The examiners were made aware of the purpose and methods of the study at the start of the first trial. The examiners were asked to take measurements using both the accepted standard method of goniometry and a novel method that uses innovative landmarks. The examiners were given a ten-minute instructional overview of the novel method; no instructional overview was given for the standard method as students had previously completed two semesters of lab training involving the assessment of range of motion measurements with goniometers. Both standard and novel measurements were taken using a 6-inch rulongmeter with 360-degree head; all measurements were measured in one-degree increments.

For standard shoulder flexion, the following anatomical landmarks were used: axis at greater tuberosity of humerus, stationary arm parallel to midline of thorax, and moving arm in line with lateral epicondyle of humerus. For standard shoulder abduction, the following anatomical landmarks were used: axis at axilla, stationary arm parallel with midline of thorax, and moving arm in line with lateral epicondyle of humerus. For novel shoulder flexion, the axis was situated in the axilla, the movement arm on the posterior aspect of the humerus, and the stationary arm on the lateral aspect of the midline of thorax (Figure 1). For novel shoulder abduction, the goniometer axis was situated on the lateral aspect of the axilla, the stationary arm on lateral aspect of the midline of the thorax, and movement arm on the medial surface of the humerus (Figure 2). For novel measurements, the examiner applied pressure to goniometer until firm end feel of soft tissue was found.

Study participants received an instructional handout of the warmup routine to be performed prior to shoulder active range of motion measurements. The routine consisted of 10 repetitions of shoulder pendulums in bilateral directions on each arm and 30 seconds forward and backwards arm circles (Figure 3). Shoulder measurements were taken with the participants in a standing position. Participants were asked to actively perform one repetition of shoulder flexion and abduction to end range; at end range, the examiner applied the goniometer to read the measurement value. After each measurement was taken, the examiners were responsible for recording values into a data table. Active shoulder flexion and abduction was performed on left and right shoulders using both the standard and novel goniometer methods. Measurements were taken on 22 participants by 22 secondyear physical therapy students. In two weeks, the participants returned, and the same examiners completed the measurements again following the same parameters. Prior to the start of the second trial the examiners were again instructed in how to perform the novel method of measurement. All participants and examiners were randomly assigned a number for data analysis.

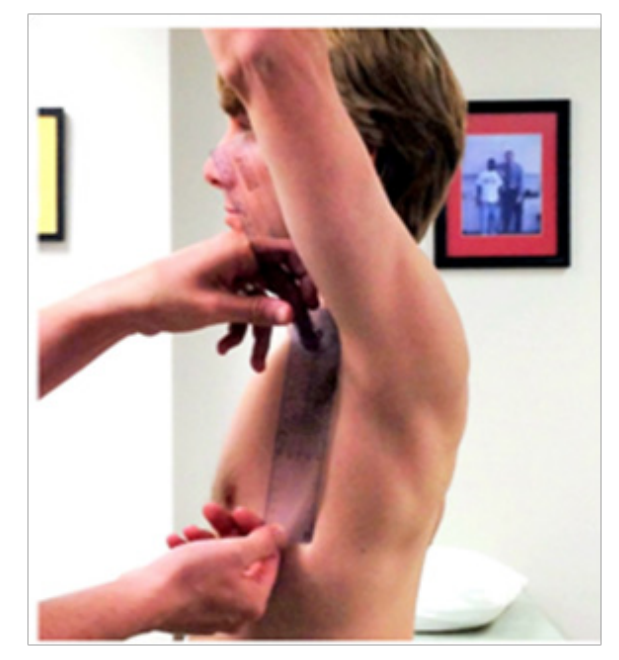

Figure I Novel method for measuring shoulder flexion.

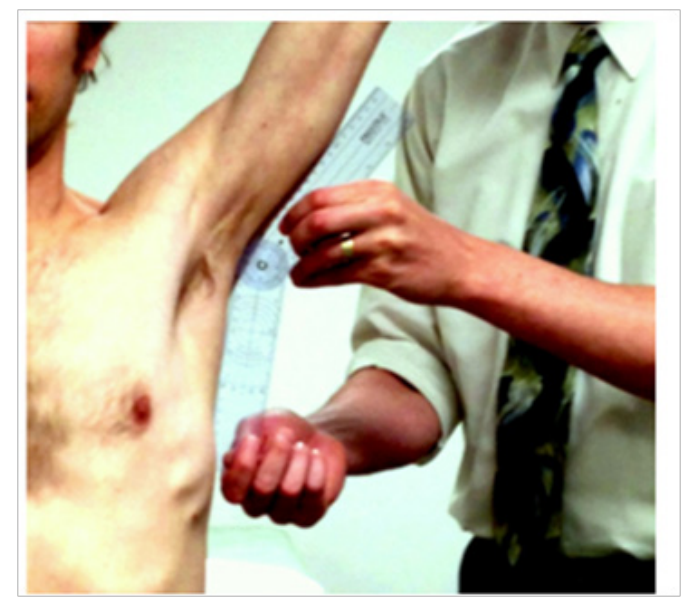

Figure 2 Novel method for measuring shoulder abduction.

$$
\begin{aligned}
& \text { Warm-Up Routine } \\
& 10 \text { repetitions of shoulder } \\
& \text { pendulums in both } \\
& \text { directions on each arm }
\end{aligned}
$$

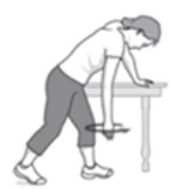

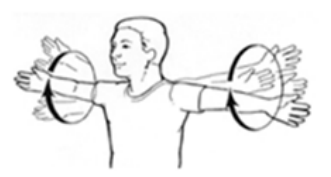

Figure 3 Instructional handout. 


\section{Statistical analysis}

Statistical analysis of the data was completed using IBM SPSS Version 25. Means and standard deviations for continuous data and frequency and percent for categorical variables were calculated for demographics. Reliability analysis was completed to find intraclass correlation coefficient (ICC) values for both intrarater and inter-rater reliability. Analysis was completed for left shoulder flexion, right shoulder flexion, left shoulder abduction, and right shoulder abduction; both the standard measurement and novel measurement data values were analyzed for each of these motions. Inter-rater reliability was found using two-way random/ consistent variance; intra-rater reliability was found using two-way mixed/absolute variance. ICC values were classified as poor $(<0.5)$, moderate (0.5-0.75), good (0.75-.90), or excellent $(>0.90)$. After analysis was completed, ranges of ICC values were found for each data set. Standard and novel ICC values were compared to determine any statistical difference between the two methods. ICC values were also compared to those in previously published goniometry studies.

\section{Results}

Demographic information of the study sample is summarized in Table 1. Data was analyzed for 44 shoulders (22 right, 22 left) from 22 participants. Participants included 13 males and 9 females with an age range of 23-43. Due to time constraints, 4 subjects and 4 examiners were unable to complete the second trial of the study.

The intra class correlation coefficient range for inter-rater and intra-rater reliability for the standard and novel goniometric measures are presented in Table 2 . Inter-rater reliability ICCs for the standard method ranged -.288 to 0.80 . For the novel method inter-rater reliability ICCs ranged from -.178 to .844 . Intra-rater reliability ICCs for the standard method ranged -.276 to .824 . For the novel method intra-rater reliability ICCs ranged from -.043 to .833 . ICC values were within poor $(\mathrm{ICC}<0.5)$ to moderate (ICC $0.5-0.75$ ) reliability ranges among the majority of examiners in both inter-rater and intra-rater reliability for both methods Table 3 .

Table I Demographics( $\mathrm{n}=22$ participants $)$

\begin{tabular}{ll}
\hline Characteristic & Mean(SD) \\
\hline Age(years) & \\
Subjects & $27.23(5.28)$ \\
Examiners & $26.95(5.36)$ \\
Sex & \\
Males & $13(59 \%)$ \\
Females & $9(41 \%)$ \\
\hline
\end{tabular}

Table 2 Intra-rater and inter-rater reliability for shoulder range of motion

\begin{tabular}{lll}
\hline Inter-rater reliability & $\begin{array}{l}\text { Standard ICC } \\
\text { range }\end{array}$ & $\begin{array}{l}\text { Novel ICC } \\
\text { range }\end{array}$ \\
\hline Rovement & -0.983 & -0.884 \\
Right flexion & $.175-.804$ & -0.819 \\
Left flexion & -0.963 & $.003-.831$ \\
Left abduction & -1.067 & -0.99 \\
Intra-rater Reliability & Standard ICC & Novel ICC \\
\hline range & range \\
\hline Right flexion & -0.961 & -0.796 \\
Right abduction & $.171-.824$ & $.133-.820$ \\
Left flexion & $.037-.766$ & $.179-.833$ \\
Left abduction & $.221-.722$ & $.236-.733$ \\
\hline
\end{tabular}

Table 3 Reliability analysis

\begin{tabular}{|c|c|c|c|c|c|c|c|c|}
\hline \multicolumn{9}{|c|}{ Inter-rater reliability } \\
\hline & \multicolumn{2}{|c|}{$\mathbf{R}$ flexion } & \multicolumn{2}{|c|}{ R abduction } & \multicolumn{2}{|c|}{ L flexion } & \multicolumn{2}{|c|}{ L abduction } \\
\hline & Std & New & Std & New & Std & New & Std & New \\
\hline Poor reliability & |4(77\%) & $12(67 \%)$ & $7(37 \%)$ & $9(47 \%)$ & $9(50 \%)$ & $13(72 \%)$ & $12(63 \%)$ & $9(47 \%)$ \\
\hline Moderate reliability & $4(22 \%)$ & $4(22 \%)$ & $9(47 \%)$ & $9(47 \%)$ & $8(44 \%)$ & $4(22 \%)$ & $6(32 \%)$ & $9(47 \%)$ \\
\hline Good reliability & $x$ & $2(11 \%)$ & $3(16 \%)$ & $\mathrm{I}(5 \%)$ & $\mathrm{I}(5 \%)$ & $\mathrm{I}(5 \%)$ & $\mathrm{I}(5 \%)$ & $\mathrm{I}(5 \%)$ \\
\hline Excellent reliability & $x$ & $x$ & $x$ & $x$ & $x$ & $x$ & $x$ & $x$ \\
\hline \multicolumn{9}{|c|}{ Intra-rater reliability } \\
\hline & \multicolumn{2}{|c|}{$R$ flexion } & \multicolumn{2}{|c|}{$\mathbf{R}$ abduction } & \multicolumn{2}{|c|}{ L flexion } & \multicolumn{2}{|c|}{ L abduction } \\
\hline & Std & New & Std & New & Std & New & Std & New \\
\hline Poor reliability & $15-83 \%$ & $15-83 \%$ & $7(39 \%)$ & $7(39 \%)$ & $7(41 \%)$ & $10(59 \%)$ & $10(55 \%)$ & $9(55 \%)$ \\
\hline Moderate reliability & $3(17 \%)$ & $2(11 \%)$ & $9(50 \%)$ & $9(50 \%)$ & $10(59 \%)$ & $7(41 \%)$ & $7(41 \%)$ & $7(39 \%)$ \\
\hline Good reliability & $x$ & $\mathrm{I}(5 \%)$ & $2(11 \%)$ & $2(11 \%)$ & $x$ & $x$ & $\mathrm{I}(5 \%)$ & $2(11 \%)$ \\
\hline Excellent reliability & $x$ & $x$ & $x$ & $x$ & $x$ & $x$ & $x$ & $x$ \\
\hline
\end{tabular}




\section{Discussion}

This research was a continuation of a previous study by Irwin et al. ${ }^{3}$ which sought to examine the current established method of goniometric measurement techniques for determining shoulder range of motion and compare its reliability to a novel method of goniometric measurement using innovative landmarks. The original study consisted of 2 examiners who were practicing clinicians and 22 volunteer patients. The procedures and shoulder motions measured were the same across both studies. The follow-up study was performed with the intention of including more examiners to increase the power of the expected results, however the outcomes of the original study showed better results regarding the reliability of the novel method versus the established method. The difference may be explained by the fact that the original study used experienced clinicians and the follow-up study relied on examiners that were selected from a cohort of $2^{\text {nd }}$ year DPT students at the beginning of their $5^{\text {th }}$ of 9 semesters of study, and after they had completed two semesters of lab training involving the assessment of range of motion measurements with goniometers.

The results revealed that the students' reliability for the standard method was significantly worse than those of practicing clinicians based on previously published evidence. Previous research comparing the universal goniometer to a virtual goniometer when performing shoulder range of motion found high validity and ICC values $>.80$ for both measurement methods. ${ }^{3}$ These results indicate that shoulder goniometry, using the standard method, is an accurate clinical tool with strong intra and inter-rater reliability; this differs significantly from the results of our study. Another published study compared the accuracy of universal goniometer to a digital inclinometer when measuring shoulder range of motion. Both methods produced ICC values $>.95$, which indicates excellent reliability. ${ }^{4}$ However, the study did find that the mean inclinometer measurements were higher than the universal goniometer. Like the results of our study, this would indicate that clinicians should be consistent with the measurement instrument and specify the instrument used in their documentation in order to accurately track patient progress.

Considering that the clinicians examined in the published data have actual clinical experience, it is not surprising that they perform goniometry more consistently than students. However, if the goal of a DPT education is to produce entry-level clinicians at graduation, then it would be expected that the students would demonstrate proficiency in this skill.

The limitations of this study could have influenced the less than desirable data results. The subjects were gathered via a convenience sample of only Georgia State University physical therapy students, who all had the same training on performing goniometer measurements. During the data collection trials, there was limited time to adequately instruct and practice the novel method prior to beginning the data collection. There was limited supervision of examiners and subjects during the trials, which could have led to examiners incorrectly or hastily performing measurements. Due to the time constraints and lack of personal investment, not all examiners took the time to be precise with all measurements. Also, due to conflicts in scheduling, not all subjects from trial one were able to be present for trial two data collection. The decline in trial two subjects decreased the experimental sample size and limited the number of complete data sets that could be analyzed for intra-rater and inter-rater reliability.

Due to the difference in positional placement for the two methods, the novel method produced range of motion values that were significantly lower than the standard measurement method. This large difference in values would prevent the two methods from being used interchangeably in the clinical setting. The clinician would be required to consistently use either the novel or standard method in order to track patient progress. Additionally, the novel method does not have normalized range of motion values for each joint therefore clinicians would not be able to compare novel measurements with the standard range of motion norms.

\section{Conclusion}

The results of the study suggest that $2^{\text {nd }}$ Year Doctor of Physical Therapy students are notably less reliable in performing goniometric measurements for active shoulder range of motion than are licensed physical therapists. This may indicate the need for further instruction and increased practice in developing the skill of goniometric measurement. Further research must be done to investigate the reliability and validity of Doctor of Physical Therapy students in assessing range of motion at the end of their $3^{\text {rd }}$ year curriculum, after increased exposure to the clinical setting and having ample opportunities to apply the skill of goniometry measurements.

\section{Funding}

None.

\section{Acknowledgments}

None.

\section{Conflicts of interest}

The authors declare no conflicts of interest.

\section{References}

1. Kolber MJ, Hanney WJ. The reliability and concurrent validity of shoulder mobility measurements using a digital inclinometer and goniometer: a technical report. Int J Sports Phys Th. 2012;7(3):306-313.

2. Diane U Jette, Alicia Bertoni, Renee Coots, et al. Clinical instructors' perceptions of behaviors that comprise entry-level clinical performance in physical therapy students: a qualitative study. Physical Therapy, 2007;87(7):833-843.

3. Irwin J, Richards KM, Johnson MS, et al. An attempt to improve accuracy and reliability of shoulder active range of motion goniometric measurement: a randomized controlled trial. Int J Orthop Stud. 2017:1(1)

4. Shishani Y, Flocken J, Gobezie R. What Is the accuracy of shoulder range of motion measurements on physical exam? Open J Orthop. 2015;5:264 276. 